\title{
Pandemic Dreams: Network Analysis of Dream Content During the COVID-19 Lockdown
}

\section{OPEN ACCESS}

Edited by:

Antonino Raffone,

Sapienza University of Rome, Italy

Reviewed by:

Tore Nielsen,

Université de Montréal, Canada

Michelle Carr,

Swansea University, United Kingdom

Maurizio Gorgoni,

Sapienza University of Rome, Italy

*Correspondence:

Anu-Katriina Pesonen

anukatriina.pesonen@helsinki.fi

tThese authors have contributed equally to this work and share last authorship

Specialty section:

This article was submitted to

Consciousness Research,

a section of the journal

Frontiers in Psychology

Received: 19 June 2020

Accepted: 04 September 2020

Published: 01 October 2020

Citation:

Pesonen A-K, Lipsanen J, Halonen R, Elovainio M, Sandman N, Mäkelä J-M, Antila M, Béchard D,

Ollila HM and Kuula L (2020)

Pandemic Dreams: Network Analysis of Dream Content During the COVID-19 Lockdown.

Front. Psychol. 11:573961. doi: 10.3389/fpsyg.2020.573961

\begin{abstract}
Anu-Katriina Pesonen ${ }^{*}$, Jari Lipsanen², Risto Halonen', Marko Elovainio', Nils Sandman ${ }^{3,4}$, Juha-Matti Mäkelä ${ }^{1}$, Minea Antila ${ }^{1}$, Deni Béchard ${ }^{5}$, Hanna M. Ollila ${ }^{6,7,8,9+}$ and Liisa Kuula ${ }^{1}$

${ }^{1}$ Sleepwell Research Program, Faculty of Medicine, University of Helsinki, Helsinki, Finland, ${ }^{2}$ Department of Psychology and Logopedics, Faculty of Medicine, University of Helsinki, Helsinki, Finland, ${ }^{3}$ Department of Psychology and Speech-Language Pathology, University of Turku, Turku, Finland, ${ }^{4}$ Department of Social Psychology, University of Helsinki, Helsinki, Finland, ${ }^{5}$ Visiting Researcher, Institute for Molecular Medicine Finland (FIMM), University of Helsinki, Helsinki, Finland, ${ }^{6}$ Department of Psychiatry and Behavioral Sciences, Stanford University, Palo Alto, CA, United States, ${ }^{7}$ Center for Genomic Medicine, Massachusetts General Hospital, Boston, MA, United States, ${ }^{8}$ Program in Medical and Population Genetics, Broad Institute, Cambridge, MA, United States, ${ }^{9}$ Institute for Molecular Medicine Finland (FIMM), University of Helsinki, Helsinki, Finland
\end{abstract}

We used crowdsourcing (CS) to examine how COVID-19 lockdown affects the content of dreams and nightmares. The CS took place on the sixth week of the lockdown. Over the course of 1 week, 4,275 respondents (mean age 43, SD = 14 years) assessed their sleep, and 811 reported their dream content. Overall, respondents slept substantially more (54.2\%) but reported an average increase of awakenings (28.6\%) and nightmares (26\%) from the pre-pandemic situation. We transcribed the content of the dreams into word lists and performed unsupervised computational network and cluster analysis of word associations, which suggested 33 dream clusters including 20 bad dream clusters, of which 55\% were pandemic-specific (e.g., Disease Management, Disregard of Distancing, Elderly in Trouble). The dream-association networks were more accentuated for those who reported an increase in perceived stress. This CS survey on dream-association networks and pandemic stress introduces novel, collectively shared COVID-19 bad dream contents.

Keywords: dream, sleep, crowdsourcing, cluster, network analysis, COVID-19, nightmare, stress

\section{INTRODUCTION}

On March 11, 2020, the World Health Organization announced that COVID-19 is a pandemic (World Health Organization, 2020). Significant events and threatening situations change the way people sleep and dream, and pandemics are no exception (Nielsen et al., 2006; Hartmann and Brezler, 2008; Sandman et al., 2013; Cenat et al., 2020). While sporadic changes in sleep and dreaming are normal, and sleep naturally responds to environmental fluctuation, extreme factors and traumatic experiences can lead to severe changes in sleep patterns, including altered dream content or more nightmares. An increase in nightmares has been previously observed with regard to wars, terrorist attacks, and during earlier pandemics or infectious diseases (Nielsen et al., 2006; Hartmann and Brezler, 2008; Sandman et al., 2013). Similarly, the COVID-19 pandemic poses a threat to health and well-being, causes worries and fears, alters behavior and everyday life, and has a lasting impact on how we perceive the world (Zhou et al., 2020). Thus far, preliminary reports suggest that poor-quality sleep and insomnia-related symptoms have indeed increased in 
the general population (Casagrande et al., 2020; Huang and Zhao, 2020; Xiao et al., 2020); however, the impact of the pandemic on the content of dreams and nightmares remains unexplored. Seizing on the opportunity and collecting dream content during a carefully selected time window during the COVID-19 lockdown allowed for a unique natural experiment to study dreams and nightmares.

Indeed, dreams reflect waking consciousness by spontaneously incorporating daytime experience (Koulack et al., 1985; Stickgold et al., 2000; Nielsen et al., 2004; Malinowski and Horton, 2014; Solomonova et al., 2015). Evidence converges to suggest that the incorporation of experience into dreams is characterized by a circaseptan process, such that dreams include events from both the preceding day (day-residue effect) and a week earlier (dream-lag effect) (Nielsen et al., 2004). In order to understand whether the stress of the pandemic influences dreams and results in collectively shared dream content (i.e., whether shared experiences, such as COVID-19 and the lockdown, produce similar dreams), it is crucial to solicit the dream reports during a very short timeframe.

Nightmares are a special case of dreaming. They can be defined as repeated occurrences of extended, extremely dysphoric, and well-remembered dreams that usually involve threats to survival, security, or physical integrity (American Psychiatric Association, 2013). Current definitions acknowledge that many nightmares are not followed by an awakening (American Psychiatric Association, 2013). While occasional nightmares are common and harmless, frequent nightmares have been associated with other sleep problems (Zadra and Donderi, 2000), symptoms of depression (Li et al., 2010), and even increased risk of suicide (Russell et al., 2019). Idiopathic nightmares can contain events and themes that reflect daytime experiences, but they are rarely repetitive or replicate events accurately (Gieselmann et al., 2019). Post-traumatic nightmares, on the other hand, have a clear relation to previously experienced traumatic events and may even replicate parts of the trauma in detail (Sandman et al., 2013; Miller et al., 2017). The COVID-19 pandemic and the lockdown had potential to generate both types of nightmares, depending on the circumstances of the dreamer.

The current study used a crowdsourcing (CS) method to investigate how COVID-19 lockdown is associated with selfreported changes in perceived stress and increases in nightmares or deviations in dream content. We took the opportunity to ask respondents to describe the content of their dreams during lockdown and then applied computational network and cluster analyses to find clustered word-association chains commonly occurring in the data. In keeping with the dream continuity hypothesis (Schredl and Hofmann, 2003), we expected dreams to exhibit novel thematic categories specifically related to the current pandemic lockdown.

\section{RESULTS}

\section{Sample Characteristics}

There were 4,275 citizens who participated in the CS (mean age $42.6, \mathrm{SD}=13.7$ years, range $10-99,79 \%$ women, $19 \%$ men, $2 \%$ other/unspecified) over the course of 1 week, and 811 respondents reported their dream content. The age distribution of the participants is shown in Table 1. The majority (57.5\%) were employed and worked from home, and the remainder had the following distribution: $12 \%$ working outside the home; $10.2 \%$ students; $8.6 \%$ retired; $8.7 \%$ unemployed/laid off; and $3.2 \%$ other. Of the participants, $24.8 \%$ lived in a single-person household, $35.3 \%$ lived with their spouse, $34.5 \%$ lived in a household with children, $2.8 \%$ lived in a shared household, and $2.6 \%$ did not specify.

\section{Perceived Stress and Sleep During the Lockdown}

Perceived stress levels increased in the majority (56\%) of respondents [high increase in 767 (18.0\%), whereas 1,620 (38.0\%) reported a modest increase]. A total of 933 (21.9\%) respondents reported unchanged, and $943(22.1 \%)$ reported lowered stress. Stress levels increased in more females than males $(P<0.001)$. The pandemic's impact on sleep patterns was assessed in terms of sleep duration, sleep latency, awakenings, the regularity of the sleep rhythm, and the frequency of nightmares (Table 2). Females were more likely than males to report increased sleep duration and more frequent nightmares $(P<0.001$ and $P<0.001$, respectively).

An increase in perceived stress levels was associated with progressively shorter sleep duration $\left(r_{\mathrm{s}}=0.26, P<0.001\right)$, prolonged sleep latency $\left(r_{\mathrm{s}}=0.37, P<0.001\right)$, more frequent nightly awakenings $\left(r_{\mathrm{s}}=0.43, P<0.001\right)$, increasingly irregular sleep rhythms $\left(r_{\mathrm{s}}=0.182, P<0.001\right)$, and more frequent nightmares $\left(r_{\mathrm{s}}=0.29, P<0.001\right)$. Figure 1 displays the prevalence of sleep disturbances in relation to respondents' perceived levels of stress. Increased disturbances

TABLE 1 | Age distribution of the sample.

\begin{tabular}{lcr}
\hline Years & $\boldsymbol{N}$ & $\%$ \\
\hline$<20$ & 75 & 2.2 \\
$20-34$ & 1051 & 30.1 \\
$35-49$ & 1273 & 36.5 \\
$50-64$ & 824 & 23.6 \\
$\geq 65$ & 264 & 7.6
\end{tabular}

788 respondents did not report age.

TABLE 2 | Change in sleep patterns from pre-COVID-19 to lockdown.

\begin{tabular}{lccccc}
\hline & $\begin{array}{c}\text { Sleep } \\
\text { duration }\end{array}$ & \multicolumn{2}{c}{$\begin{array}{c}\text { Sleep Awakenings } \\
\text { latency }\end{array}$} & \multicolumn{1}{c}{$\begin{array}{c}\text { Sleep } \\
\text { regularity }\end{array}$} & Nightmares \\
\hline Decreased (\%) & 16.6 & 13.0 & 11.7 & 16.3 & 3.5 \\
No change (\%) & 29.1 & 77.4 & 59.7 & 53.8 & 70.4 \\
Increased (\%) & 54.2 & 9.6 & 28.6 & 29.9 & 26.0 \\
\hline
\end{tabular}

Lockdown-associated sleep changes per respondent

$\begin{array}{lcclll}\text { Number of sleep items } & 1 & 2 & 3 & 4 & 5 \\ \text { Complaints (\%) } & 24.0 & 15.6 & 8.7 & 3.1 & 0.6 \\ \text { Improvements (\%) } & 32.1 & 20.8 & 8.7 & 2.6 & 0.4\end{array}$




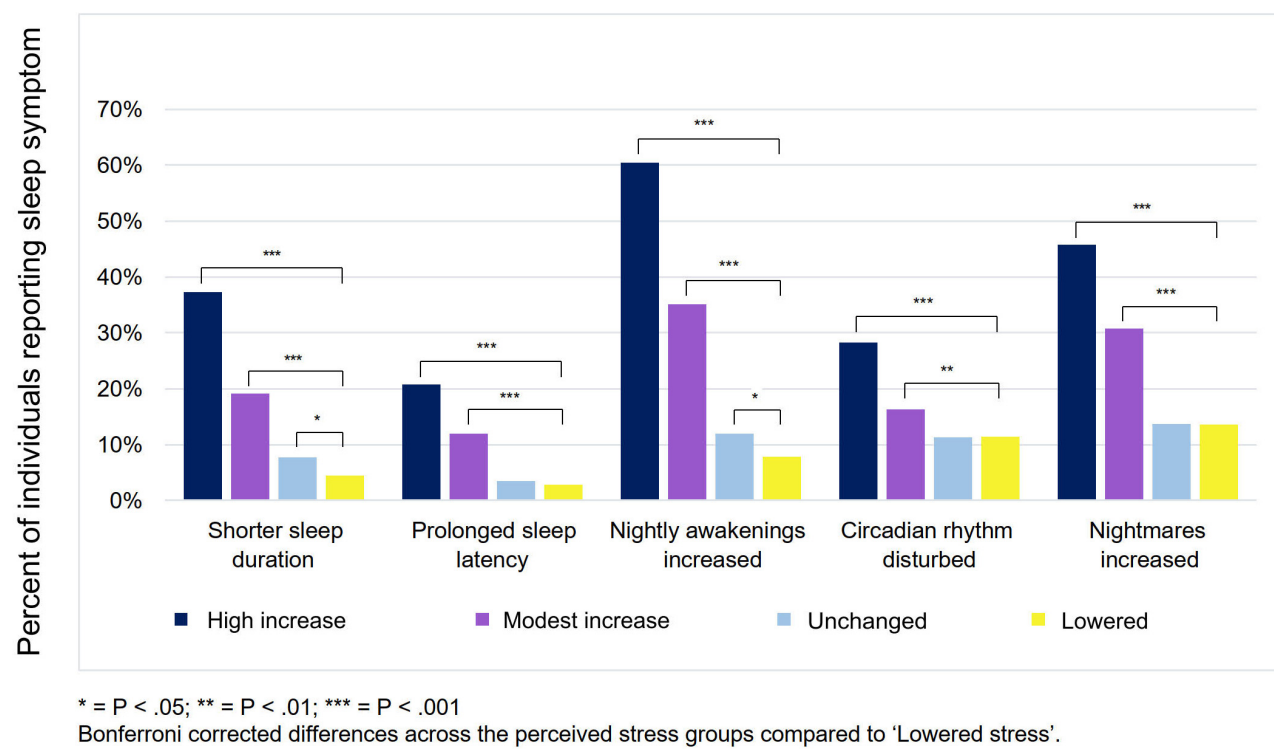

FIGURE 1 | The percentage of individuals reporting worsening sleep (shorter sleep duration, longer sleep latency, more awakenings, disturbed circadian rhythm, and more nightmares) according to their experience of COVID-19-related stress.

were concentrated primarily in those who reported a high increase in stress; their sleep worsened linearly with the increase in perceived stress. The most prominent increases were for nightly awakenings (60.5\%) and nightmares (45.8\%) in the groups with the highest perceived stress $(P<0.001)$.

Regarding beneficial effects, those with lowered stress levels during the lockdown reported longer sleep duration $(73.9 \%)$ and more regular sleep rhythm (44.9\%) compared to their prepandemic sleep.

\section{Dream Content Frequencies and Networks}

Dreams were reported by different frequencies in different stress groups (high increase in stress $=27.4 .5 \%$; modest increase in stress $=20.5 \%$; stress unchanged $=13.0 \%$; stress lowered $=17.8 \%), \chi^{2}=58.427, P<0.001$. Age was not associated with the likelihood of reporting dreams $(P=0.087)$. Females were more likely to report dream content than males $\left(\chi^{2}=72.423, P<0.001 ; 21.6\right.$ and $9.6 \%$, respectively). In addition, working status differed between dream reporters and non-reporters $\left(\chi^{2}=23.022, P<0.001\right)$. Students and retired persons were more likely to deliver a dream report, whereas those working remotely were less likely (Supplementary Figure S1).

Figure 2 displays the most frequent words in the dream report data: column 1 for "stress increased" (words: coronavirus, death, work; modest or high increase responses combined) and column 2 for "Stress-unchanged/lowered" (words: crowd, friend, coronavirus).

Next, we created an unsupervised network analysis in which word associations are displayed in a correlation network. Unsupervised cluster analysis suggested 33 dream clusters in all of the groups combined, 27 in the "stress increased" group, and 11 in the "stress unchanged/lowered" group. Only words with a minimum of five occurrences and correlations $>0.2$ were retained in the final network models.

Figure 3 displays the dream clusters in groups based on stress level. After performing exploratory computation, we qualitatively analyzed the content of each dream cluster and labeled it accordingly (Table 3 ).

A subset of the dreams were highly pandemic-specific. For example, cluster 2 (all respondents) comprises threedimensional associations between mistake-hug, hug-handshake, handshake-restriction, handshake-distancing, distancingdisregard, distancing-crowd, crowd-restriction, and crowd-party, and we labeled it "Disregard of Distancing" and rated it as pandemic-specific. As another example, cluster 1 (all respondents) included the following word associations: returnmissing, missing-suitcase, missing-bus, bus-overcrowding, suitcase-lost, lost-city, city-street. While some of the clustered words referred to idiopathic nightmare content, the association with overcrowding made it pandemic-specific. We labeled it “Travel Difficulties, Overcrowding" (Table 3). As an example of overlapping clusters, cluster 1 overlapped with cluster 17 ("Burglary") through the word return in both clusters (return-home, home-burglary, home-obstacle).

The number and content of identified clusters varied according to the stress level. While most of the clusters' contents related to themes that could be clearly identified through their association networks, a few of the COVID-19-related clusters included large association networks of different pandemicassociated threats and landscapes.

Figure 4 shows that the data resulted in the following words that were most central, i.e., most frequently connected with other words independently of each other (degree of centrality): surgery, soldier, running (in the group of all respondents); surgery, doctor, 

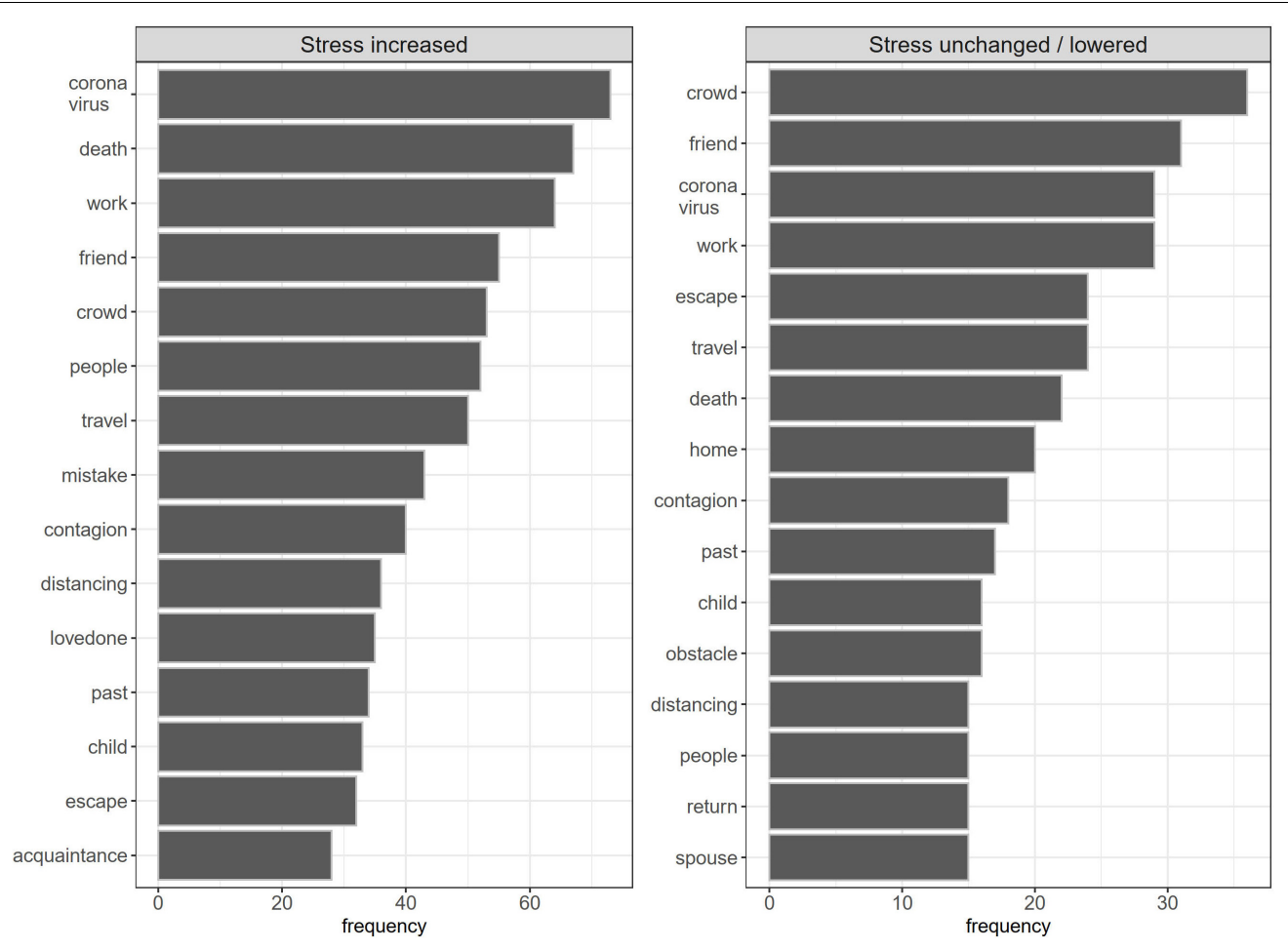

FIGURE 2 | The appearance frequency of different words in dream reports according to the experience of COVID-19-related stress (left panel: stress increased; right panel: stress unchanged/lowered).

help (in the group where stress was increased); and darkness, return, $\operatorname{dog}$ (in the group where stress was unchanged/lowered). The three most frequent node words in terms of betweenness (i.e., the number of times word $\mathrm{X}$ lies on the shortest path between words $\mathrm{Y}$ and $\mathrm{Z}$ ) were surgery, running, hospital (in the group of all respondents), surgery, running, bus (stress increased group), and darkness, return, horror (stress unchanged/lowered group). The three most frequent words in terms of closeness indicators were surgery, running, hospital (in the group of all respondents); surgery, running, hospital (stress increased group); and darkness, horror, return (stress unchanged/lowered group). The "stress increased" group had pandemic-specific network nodes, whereas nodes in the "stress unchanged/lowered" group reflected conventional nightmare/bad dream content.

\section{DISCUSSION}

We explored sleep patterns and pandemic dream content during the sixth week of the lockdown. Instead of traditional a priori-defined thematic content analysis of dreams, we used a computational exploratory, unsupervised network analysis in which single words were associated to create a network of dream content (Pons and Latapy, 2005). These networks were clustered based on the association networks around single words. The resulting dream clusters allowed identification of novel and commonly shared dream content during a time-restricted lockdown period.
The impact of lockdown on sleep quality was twofold. The majority of the respondents reported having a longer sleep duration, and almost a third reported having more regular sleep rhythms during the lockdown compared to the pre-lockdown situation, likely reflecting alleviated pressure in scheduling due to working from home. Lockdown-related sleep complaints were also common: almost a third reported more frequent awakenings at night, and more than a quarter of the respondents also had an increase in nightmares. As could be expected (Nielsen et al., 2006; Nielsen and Levin, 2007; Robert and Zadra, 2014), we observed a larger increase in the frequency of nightmares among respondents who also reported elevated stress during the lockdown, although no causality can be inferred.

Our analysis of dream content revealed typical, idiopathic nightmares/bad dreams (e.g., falling, being chased, being late, and the deaths of loved ones) (Robert and Zadra, 2014), comparable to nightmares reported in a very similarly aged population-representative sample in 2010 (mean age $=46$, SD = 16 years) (Schredl, 2010). Health-related concerns represented $12 \%$ of the distressing dream content in the general population (Robert and Zadra, 2014). In the current study, we identified 33 different dream clusters (in all respondents), of which 20 were evaluated as having distressing dream content, and of these, 55\% were pandemic-specific. Themes such as failures in social distancing, coronavirus contagion, personal protective equipment (PPE), dystopia, and apocalypse were rated as pandemic-specific. Most of these were confounded with established idiopathic dream categories (e.g., failure, death, 

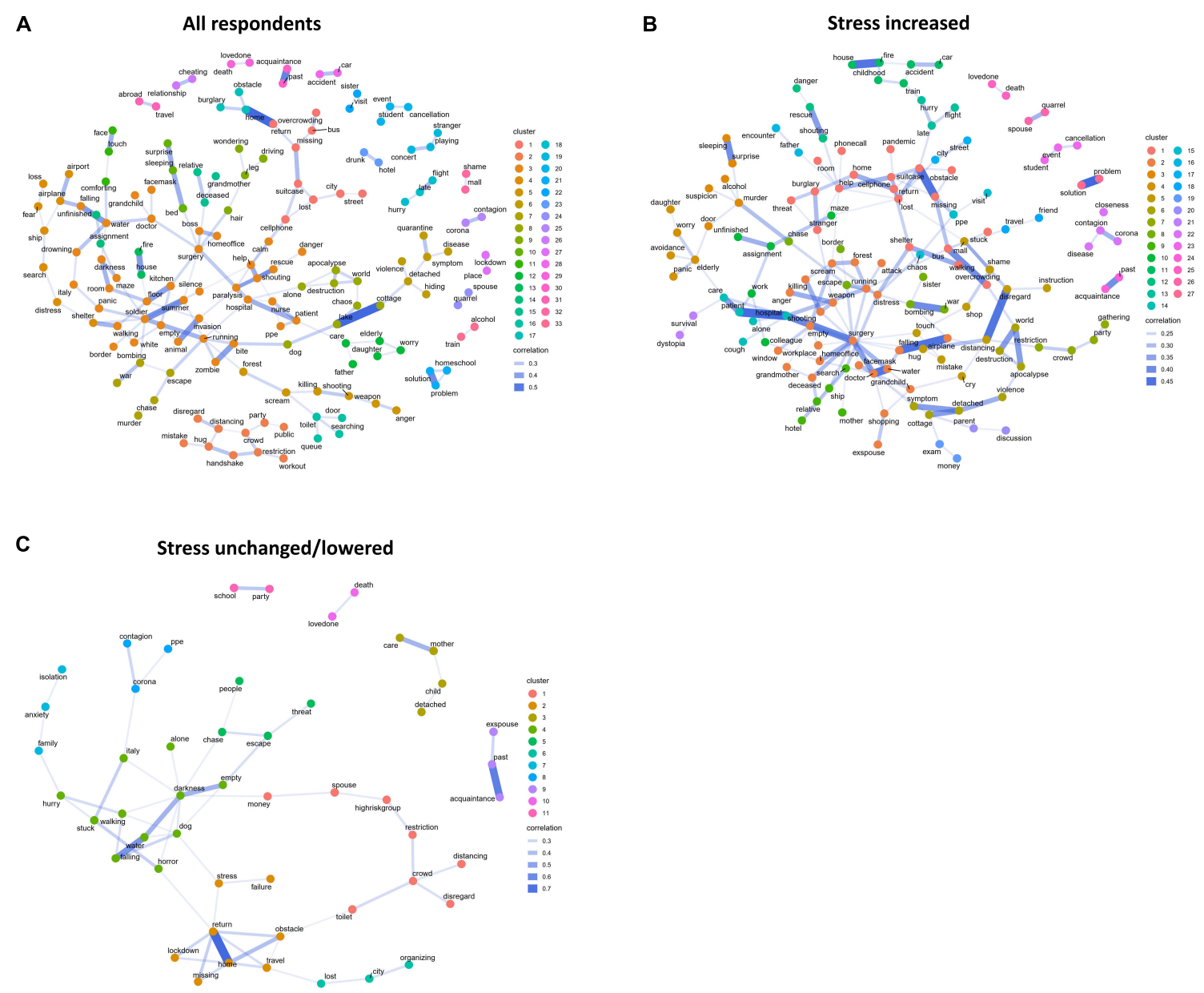

FIGURE 3 | Dream content clusters according to perceived stress in panel (A) all respondents, panel (B) in respondents with stress increased, panel (C) in respondents with stress unchanged/lowered. Colors refer to computational clusters, where dots/words with similar color belong to the same cluster. The level of strength of association between two words is illustrated with a stronger line between them. Lines indicate a statistically significant association.

worry), but the dream imagery was specific to the current pandemic situation.

Sleep enables offline processing of recent memory traces (Peigneux et al., 2003). Sleeping enhances newly acquired memories through a physiological reactivation of brain areas recruited during learning (Klinzing et al., 2019), as activation in these specific brain regions is known to correlate with particular dream content (Siclari et al., 2017). Fear-related dream imagery (often experienced in bad dreams, idiopathic nightmares, and post-traumatic nightmares) associated with emotional arousal can equally serve to extinction of fear memories (Nielsen and Levin, 2007) and would then assist in the emotional adaptation to the presence of COVID-19, as REM sleep plays a critical role in emotional processing (Tempesta et al., 2018). Dreams related to failures in social distancing, for example, may then help in consolidating episodic memory for new behavioral rules and routines in social situations.

Hartmann's contextualizing hypothesis proposes that nightmares serve the function of contextualizing, or finding a picture context for, an individual's predominant emotional concerns (Hartmann, 1998; Hartmann and Brezler, 2008). In line with that, our analytical approach to associative dream content networks was particularly insightful in terms of revealing pandemic-specific dream imagery, as we detected some central dream images (e.g., surgery, PPE) that may refer to fear but were contextualized in varying dream events. Overall the findings from analysis of dream content are consistent with both threat simulation theory (Revonsuo, 2000) and the dream continuity hypothesis (Schredl and Hofmann, 2003), as we identified dreams that potentially prepare the dreamer for negative events that may take place (threat simulation) and furthermore replicate those events that are observed during waking (continuity).

Comparing the dream-association networks according to perceived (daytime) stress, we found fewer repeated association networks in the "stress unchanged/lowered" group, but roughly half of the dream-association networks were still pandemicspecific, as was also the case in the "stress increased" group. The most frequent dream words were corona[virus], crowd, and death among those with increased stress levels, and crowd, friend, and corona[virus] among those with unchanged/lowered stress 
TABLE 3 | Dream cluster labels according to perceived stress.

\begin{tabular}{|c|c|c|}
\hline All respondents & Stress increased & $\begin{array}{l}\text { Stress } \\
\text { unchanged/lowered }\end{array}$ \\
\hline $\begin{array}{l}\text { 1. *Travel Difficulties, } \\
\text { overcrowding }\end{array}$ & $\begin{array}{l}\text { 1. *Travel Difficulties, } \\
\text { overcrowding }\end{array}$ & $\begin{array}{l}\text { 1. *Disregard of } \\
\text { distancing }\end{array}$ \\
\hline $\begin{array}{l}\text { 2. *Disregard of } \\
\text { distancing }\end{array}$ & $\begin{array}{l}\text { 2. *Falling } \\
\text { 3. *Murder in the night }\end{array}$ & $\begin{array}{l}\text { 2. *Obstacles in } \\
\text { returning home }\end{array}$ \\
\hline 3. *Surgery and & 4. *Elderly in trouble & 3. Care of family \\
\hline troubles & 5. *Disregard of & 4. *Falling into dark \\
\hline 4. Travel & distancing & water \\
\hline 5. *Violence with & 6. *Apocalypse & 5. *Chase-escape \\
\hline weapons & 7. *Crowd-restriction & 6. *Being lost in a city \\
\hline $\begin{array}{l}\text { 6. *Quarantine and } \\
\text { disease symptoms }\end{array}$ & $\begin{array}{l}\text { 8. *Chase-escape in } \\
\text { war and borders }\end{array}$ & $\begin{array}{l}\text { 7. *Isolation anxiety } \\
\text { 8. *Coronavirus }\end{array}$ \\
\hline 7. *War & 9. *Searching the lost & contagion \\
\hline 8. *Apocalypse & 10. *Unfinished task & 9. People from past \\
\hline 9. Body and hair & 11. *Transport accident & 10. *Death of loved one \\
\hline 10. Touch & 12. *Being in danger & 11. School party \\
\hline 11. Nightly surprise & and shouting & $4 / 9$ of mainly \\
\hline 12. *Elderly in trouble & 13. *Being late & distressing dream \\
\hline 13. *Fire & 14. *Patient care, & clusters are \\
\hline 14. *Unfinished task & cough & pandemic-specific \\
\hline 15. Deceased & 15. *Personal & $(44 \%)$ \\
\hline grandmother & protective equipment & \\
\hline 16. *Toilet and queuing & 16. Father & \\
\hline 17. *Burglary & 17. City landscape & \\
\hline 18. *Being late & 18. Travel & \\
\hline 19. Concert & 19. *Exam & \\
\hline 20. *Event cancellation & 20. Discussion with & \\
\hline 21. Homeschool & parent & \\
\hline 22. Sister visit & 21. *Dystopia & \\
\hline 23. Being drunk & 22. *Coronavirus & \\
\hline 24. *Quarrel with & contagion & \\
\hline spouse & 23. *Event cancellation & \\
\hline 25. *Coronavirus & 24. People from past & \\
\hline contagion & 25. *Death of loved one & \\
\hline \multicolumn{3}{|l|}{ 26. Cheating in } \\
\hline relationship & 26. *Quarrel with & \\
\hline 27. *Lockdown & spouse & \\
\hline 28. ${ }^{\star}$ Car accident & 27. Problem solution & \\
\hline 29. *Death of loved one & $11 / 21$ of mainly & \\
\hline 30. People from past & distressing dream & \\
\hline 31. Travel abroad & clusters are & \\
\hline 32. *Shame in public & pandemic-specific & \\
\hline place & $(52 \%)$ & \\
\hline \multicolumn{3}{|l|}{ 33. Drinking alcohol } \\
\hline \multicolumn{3}{|l|}{$11 / 20$ of mainly } \\
\hline \multicolumn{3}{|l|}{ distressing dream } \\
\hline \multicolumn{3}{|l|}{ clusters are } \\
\hline \multicolumn{3}{|l|}{ pandemic-specific } \\
\hline$(55 \%)$ & & \\
\hline
\end{tabular}

Bolded labels rated as pandemic-specific. *Bad dreams or nightmares.

levels. This would suggest that the pandemic has a substantial impact on the content of dreams independently of perceived stress. However, the available information could not distinguish between post-traumatic nightmares and the incorporation of waking events and worries into the dream context without related emotion.

A particular strength of the study is in computational analysis of the dream reports. The analyses of dream reports with unsupervised algorithms were then free from a priori defined dream categories. As another asset of this study, the CS was open only during 1 week, allowing investigation of time-bound dream content in relation to the progress of the COVID-19 lockdown. Just 10 days after the CS ended, the COVID-19 restrictions were partly lifted (for example, schools were reopened). The timebound nature of the CS is important as dreaming is thought to constitute a valuable model for the study of consciousness with implications beyond sleep (Siclari et al., 2017). This is because in normal sleep we are at least partly disconnected from environmental input and from performance of any tasks. The current approach may also be inspected from the perspective of dreams as a collectively shared consciousness during the COVID19 lockdown.

In the same vein, these interpretations are also limited by a number of methodological constraints. Dream reports are never fully accurate, and they are confounded by a number of factors: elapsed time, memories, other dreams, forgetting, and translation to a verbal narrative (Waterman et al., 1993; Windt, 2010). Some people recall very specific and visually rich dreams, whereas the dream reports of others contain only one specific dream event, such as being chased. The newspaper article on stress and nightmares published jointly with the invitation to the CS questionnaire may also, apart from inspiring respondents to report their dreams, cause systemic bias. In addition, our classification of nightmares/bad dreams was not based on subject ratings, as is typically the case; rather, our panel of judges made qualitative judgments as to whether or not dreams were distressing. The CS might have attracted more responders with sleep problems or with a fresh experience of a pandemic-specific dream, resulting in a systemic bias. We also acknowledge that the data analytic steps included human-made decisions, such as harmonizing words and expressions, and classification of dream report content as pandemic or non-pandemic. While the method we applied is completely novel in dream report analysis, it required intensive teamwork and shared decisionmaking processes. This study did not then allow for the testing of inter-rater reliability. Additionally, the groups with different stress levels were not even in terms of dream report material, as participants with increased stress reported more frequently dreams, and consequently, the data and the related association networks were wider. It is of note that while some cluster networks, especially in the low stress group, were very narrow and easy to label, some cluster networks were wide and partly unfocused. Labeling clusters is based on qualitative interpretations and coming to a consensus on the best fitting label. Yet, the process is fully transparent as all cluster data are openly presented in the Figure 3.

To sum up, we applied a network analysis to explore dream content during COVID-19 lockdown. We found several pandemic-specific dream contents and dream imagery that were associated with a variety of distressing events. A large proportion of the respondents had increased stress, which was associated with nightmares and sleep disturbances. Because sleep disturbances and nightmares are known to predict depression and a range of other mental health problems (Li et al., 2010; Sandman et al., 2015; Baglioni et al., 2016), a consequence of 


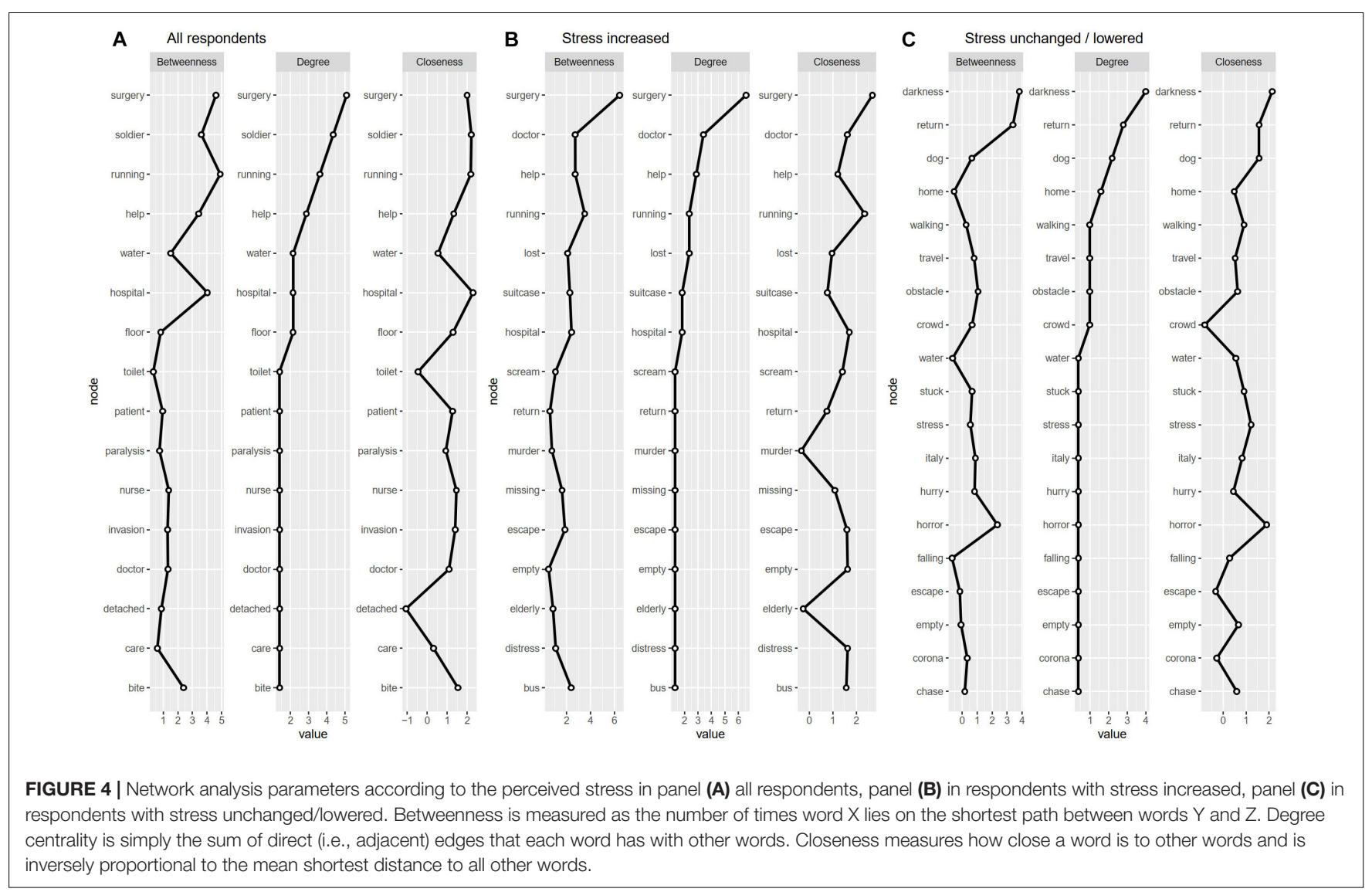

the COVID-19 pandemic may be that mental health is being impaired around the world.

\section{MATERIALS AND METHODS}

\section{Crowdsourcing}

Figure 5 presents the timeline of the pandemic onset and lockdown in Finland, as well as the timing of our CS survey on sleep. We collaborated with the most widely circulated newspaper in Finland [Helsingin Sanomat (HS), weekly reach of readers $N=2 \mathrm{M}$, average daily readers $N=339,437$ ] to generate broad participation in the very short timeframe of 1 week. The short time window maximized the situational context, which was particularly important for the dream reports. The survey was launched attached to a digital newspaper article on sleep (published April 27, 2020). On January 5, 2020, HS published a reminder that the survey is ongoing and now without paywall. The data were extracted on May 5, 2020, for the analyses. All readers were invited to respond through an internet survey, which gathered data regarding sleep during the COVID-19 lockdown. By filling in the questionnaire, the respondents also consented to the anonymized data being delivered to the Sleep and Mind Research Group at the University of Helsinki, Finland. The consent formulation was as follows: "By filling in the questionnaire, you consent to report your sleep and dreaming to Helsingin Sanomat (newspaper) and to researchers about how the pandemic lockdown has influenced your sleep. Please leave your name and contact information if your responses can be directly cited in the newspaper article. Your name will not be published, but Helsingin Sanomat requires the identity of the respondents to be known by the newspaper if they are directly cited. The newspaper office will not distribute your personal information to third parties. Responses to the questionnaire may also be used by the Sleep and Mind Research Group at the University of Helsinki. Helsingin Sanomat will only share anonymized data (without name and contact information) with the Sleep and Mind Research Group." We asked the respondents to assess how their sleep patterns had changed since the COVID-19 lockdown with regard to sleep duration, number of awakenings, sleep latency, frequency of nightmares (increased, not changed, decreased), and sleep rhythm regularity (change/no change). An evaluation of perceived stress was also grounded in how they believed their levels of stress had changed since the lockdown began (high increase, modest increase, unchanged, lowered, that were further aggregated to two groups: stress increased and stress unchanged/lowered).

\section{Treatment of the Dream Content}

The respondents were asked the following question: "Describe your dreams during the pandemic lockdown" (which included open space to write). Some respondents wrote two dreams, which were treated as separate reports. While mainstream studies of 


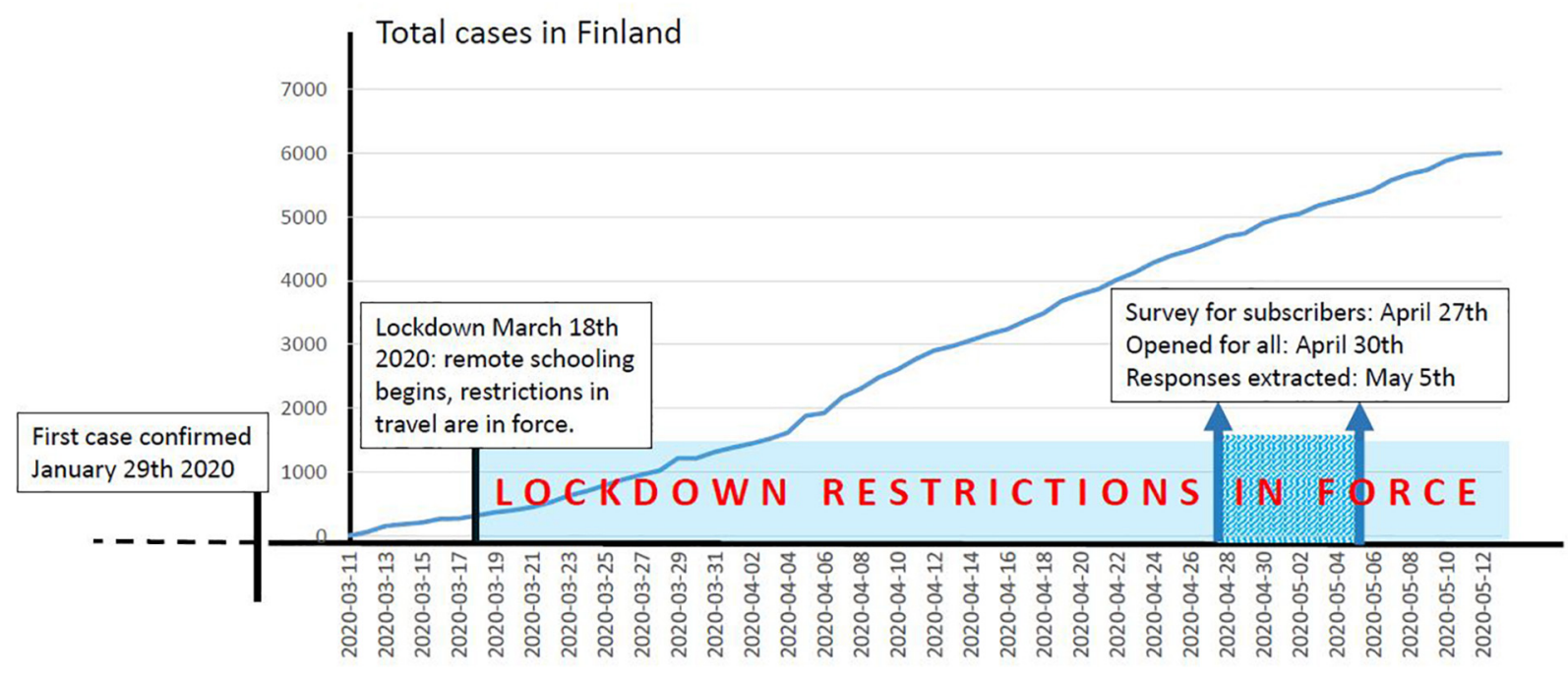

FIGURE 5 | Timing of the crowdsourcing survey from the first confirmed COVID-19 case in Finland to May 5, 2020, when the current data were extracted. Light blue indicates the period under lockdown restrictions, including remote schooling and travel restrictions within Finland and abroad. In Finland, individuals were allowed to leave their house freely and spend time outdoors. Gathering size was limited to 10. There were no restrictions relating to outdoor activities, and public transport was available. A period in darker turquoise marks the timing of the data collection.

nightmares and distressing dreams have used a priori defined thematic grids in the dream content classification (Robert and Zadra, 2014), the current study was purely computational, without any predefined dream-content grids. This method allowed an exploratory analysis of the contents of dreams that has not been used before in dream research. However, before the computational phase, the data were harmonized; for instance, dream reports were reduced to word lists containing the essential nouns in the dream report. The dream reports were first transcribed into chronological word lists for each dream separately. For example, a dream report ("I had tuberculosis and the doctor was angry with me because I did not come to see him when the first symptom appeared") was transcribed to "tuberculosis-doctor-anger-symptom." After the first draft of the transcripts was created, synonymous words and the spelling format of different words (e.g., shoe vs. shoes) were harmonized. If a word appeared twice in the dream script, it also appeared twice in the harmonized version to maintain the chronological order of the events. In addition to harmonizing the dream scripts according to the nouns, harmonizing also involved grouping some word types into categories. For example, all relatives beyond father, mother, grandfather, grandmother, daughter, and son (that were retained as such) were grouped under "relative." Recall of specific cities was grouped under "city," whereas different country names were retained. Synonymous words or expressions were replaced with one chosen word. For example, there were many different expressions for "overcrowding," which was the word that we chose in the harmonization process. The original reports were in Finnish, and translation into English required harmonization, as the vocabulary in different languages differs (Finnish: paljon ihmisiä [a lot of people]; English: crowd). Compound words in Finnish were retained as compound words in English, even when the equivalent was not linguistically correct (e.g., high-risk-group; loved-one). References to coronavirus were retained as corona, as in the original language. All dream scripts were reviewed at least twice, and all harmonization decisions were done collectively after discussion between five experts. The harmonized dream reports included 4,743 words, out of which 1,095 were unique (the respective numbers were 1,514 and 589 among stress unchanged/lowered and 3,226 and 877 among stress increased group). Note that the Finnish language is morphologically highly complex, and harmonization could not be done computationally, as has been done in English text analytics (Kettunen, 2006). We did not ask whether the respondents experienced their dream as distressing/bad, but the panel classified the dreams based on their content as bad dreams (or not) based on a shared panel view. If the resulting dream word clusters included clearly lockdown or pandemic-specific word associations, the panel judged the dream as pandemic-specific. The assessment was fully qualitative, and to ensure full transparency of the classifications, we show the word association source data and the clusters fully in Figure 3.

\section{Statistical Analyses}

We used Bonferroni-corrected one-way analysis of variance in examining sex differences in stress and sleep measures. Spearman correlations were used to study how perceived stress level was associated with self-reported sleep. Pearson $\chi^{2}$ was used to test if the relative frequency of reporting a dream differed between age, sex, and demographic status. The associations between perceived stress level and dream reports were analyzed in the following steps: (1) we divided the respondents according to their perceived stress level into "stress increased" and "stress unchanged/lowered;" (2) we calculated the most frequently used words in dream descriptions in both groups; (3) we analyzed the associations between words by tokenizing each dream with pairs 
of adjacent words (dividing each dream description into word pairs); (4) we calculated the frequency of words co-appearing and the pairwise phi correlations/distances between words (Silge and Robinson, 2016). Phi correlation is a common measure for binary correlation. The focus of the phi coefficient is how much more likely it is that either both words $\mathrm{X}$ and $\mathrm{Y}$ appear, or neither does, than that one appears without the other. In the final step (5), we illustrated these associations separately for correlations of co-appearance, creating networks for the groups with "increased stress" and "stress unchanged/lowered," and estimated both clustering (using Spinglass algorithm) of the words and degree centrality (strength) (Pons and Latapy, 2005). Spinglass algorithm is a community detection algorithm that finds groups of words that are closely connected to each other and tend to co-occur (Reichardt and Bornholdt, 2006). Of the different algorithms, it is particularly suitable for the size of the current dataset (Yang et al., 2016).

The word association networks were also analyzed using statistical indexes. Centrality represents the connectedness of a given node (words) with all the others in the network. Nodestrength centrality was defined as the sum of all associations that a given node (word) exhibits with all others in the network (the sum of the weighted number and strength of all connections of a specific node relative to all other nodes) (Freeman, 1978; Valente and Foreman, 1998). Betweenness and closeness measures are based on the shortest path length (number of connections form node to another) connecting any two nodes (words). A word with high betweenness lies along the shortest path connecting many other words in the network. Thus, the target word would have a high betweenness centrality if it appears in many shortest paths (Freeman, 1977). Closeness is calculated as the reciprocal of the sum of the length of the shortest paths between the node and all other nodes in a network; it is useful for finding the words that are best placed to influence the entire network most quickly. Thus, the more central a node is, the closer it is to all other nodes (Sabidussi, 1966). These analyses were conducted using R 3.6.1. (Pedersen, 2017).

\section{DATA AVAILABILITY STATEMENT}

The datasets presented in this article are not readily available because the crowdsourcing was performed by Helsingin Sanomat

\section{REFERENCES}

American Psychiatric Association. (2013). Diagnostic and Statistical Manual of Mental Disorders (DSM-5 $\left.{ }^{\circledR}\right)$. Washington, D.C: American Psychiatric Pub.

Baglioni, C., Nanovska, S., Regen, W., Spiegelhalder, K., Feige, B., Nissen, C., et al. (2016). Sleep and mental disorders: a meta-analysis of polysomnographic research. Psychol. Bull. 142, 969-990. doi: 10.1037/bul0000053

Casagrande, M., Favieri, F., Tambelli, R., and Forte, G. (2020). The enemy who sealed the world: effects quarantine due to the COVID-19 on sleep quality, anxiety, and psychological distress in the Italian population. Sleep Med. 75, 12-20. doi: 10.1016/j.sleep.2020.05.011

Cenat, J. M., Mukunzi, J. N., Noorishad, P. G., Rousseau, C., Derivois, D., and Bukaka, J. (2020). A systematic review of mental health programs among populations affected by the Ebola virus disease. J. Psychosom. Res. 131:109966. doi: 10.1016/j.jpsychores.2020.109966 who owns the data. Requests to access the datasets should be directed to anukatriina.pesonen@helsinki.fi.

\section{ETHICS STATEMENT}

Ethical review and approval was not required for the study on human participants in accordance with the local legislation and institutional requirements. The patients/participants provided their written informed consent to participate in this study.

\section{AUTHOR CONTRIBUTIONS}

A-KP as the PI of the study designed the study and data collection, led the dream data preparation, and manuscript writing. JL designed and conducted the network analyses and contributed to manuscript writing. $\mathrm{RH}$ and $\mathrm{ME}$ prepared the dream data, assisted in statistical analyses, and contributed to manuscript writing. NS contributed to manuscript writing and critically reviewed it. JM-M and MA prepared the dream data and critically reviewed the manuscript. DB critically reviewed the manuscript. HO contributed essentially to the manuscript writing. LK contributed essentially to each stage of the data collection and manuscript writing. All authors contributed to the article and approved the submitted version.

\section{ACKNOWLEDGMENTS}

We acknowledge science journalist Juha Merimaa for planning the CS with us and writing the article in the national newspaper.

\section{SUPPLEMENTARY MATERIAL}

The Supplementary Material for this article can be found online at: https://www.frontiersin.org/articles/10.3389/fpsyg. 2020.573961/full\#supplementary-material

Supplementary Figure 1 | Percentage of respondents delivering a dream report according to their working status.

Freeman, L. C. (1977). A set of measures of centrality based on betweenness. Sociometry 40, 35-41. doi: 10.2307/3033543

Freeman, L. C. (1978). Centrality in social networks conceptual clarification. Soc. Networks 1, 215-239. doi: 10.1016/0378-8733(78)90021-7

Gieselmann, A., Ait Aoudia, M., Carr, M., Germain, A., Gorzka, R., Holzinger, B., et al. (2019). Aetiology and treatment of nightmare disorder: state of the art and future perspectives. J. Sleep Res. 28:e12820. doi: 10.1111/jsr. 12820

Hartmann, E. (1998). Nightmare after trauma as paradigm for all dreams: a new approach to the nature and functions of dreaming. Psychiatry 61, 223-238. doi: 10.1080/00332747.1998.11024834

Hartmann, E., and Brezler, T. (2008). A systematic change in dreams after 9/11/01. Sleep 31, 213-218. doi: 10.1093/sleep/31.2.213

Huang, Y., and Zhao, N. (2020). Generalized anxiety disorder, depressive symptoms and sleep quality during COVID-19 outbreak in China: a web-based 
cross-sectional survey. Psychiatry Res. 288:112954. doi: 10.1016/j.psychres. 2020.112954

Kettunen, K. (2006). Developing an automatic linguistic truncation operator for best-match retrieval of Finnish in inflected word form text database indexes. J. Inform. Sci. 32, 465-479. doi: 10.1177/016555150606 6057

Klinzing, J. G., Niethard, N., and Born, J. (2019). Mechanisms of systems memory consolidation during sleep. Nat. Neurosci. 22, 1598-1610. doi: 10.1038/s41593019-0467-3

Koulack, D., Prevost, F., and De Koninck, J. (1985). Sleep, dreaming, and adaptation to a stressful intellectual activity. Sleep 8, 244-253. doi: 10.1093/ sleep/8.3.244

Li, S. X., Zhang, B., Li, A. M., and Wing, Y. K. (2010). Prevalence and correlates of frequent nightmares: a community-based 2-phase study. Sleep 33, 774-780. doi: 10.1093/sleep/33.6.774

Malinowski, J., and Horton, C. L. (2014). Evidence for the preferential incorporation of emotional waking-life experiences into dreams. Dreaming 24:18. doi: 10.1037/a0036017

Miller, K. E., Brownlow, J. A., Woodward, S., and Gehrman, P. R. (2017). Sleep and Dreaming in Posttraumatic Stress Disorder. Curr. Psychiatry Rep. 19::71.

Nielsen, T., and Levin, R. (2007). Nightmares: a new neurocognitive model. Sleep Med. Rev. 11, 295-310. doi: 10.1016/j.smrv.2007.03.004

Nielsen, T. A., Kuiken, D., Alain, G., Stenstrom, P., and Powell, R. A. (2004). Immediate and delayed incorporations of events into dreams: further replication and implications for dream function. J. Sleep Res. 13, 327-336. doi: 10.1111/j.1365-2869.2004.00421.x

Nielsen, T. A., Stenstrom, P., and Levin, R. (2006). Nightmare frequency as a function of age, gender, and September 11, 2001: findings from an Internet questionnaire. Dreaming 16:145. doi: 10.1037/1053-0797.16.3.145

Pedersen, T. L. (2017). Ggraph: an Implementation of Grammar of Graphics for Graphs and Networks. R package version 0. 1, 1.

Peigneux, P., Laureys, S., Fuchs, S., Destrebecqz, A., Collette, F., Delbeuck, X., et al. (2003). Learned material content and acquisition level modulate cerebral reactivation during posttraining rapid-eye-movements sleep. Neuroimage 20, 125-134. doi: 10.1016/s1053-8119(03)00278-7

Pons, P., and Latapy, M. (2005). Computing Communities in Large Networks Using Random Walks. Berlin: Springer.

Reichardt, J., and Bornholdt, S. (2006). Statistical mechanics of community detection. Physical. Rev. E 74:016110.

Revonsuo, A. (2000). The reinterpretation of dreams: an evolutionary hypothesis of the function of dreaming. Behav. Brain Sci. 23, 877-901. doi: 10.1017/ s0140525x00004015

Robert, G., and Zadra, A. (2014). Thematic and content analysis of idiopathic nightmares and bad dreams. Sleep 37, 409-417. doi: 10.5665/sleep.3426

Russell, K., Allan, S., Beattie, L., Bohan, J., MacMahon, K., and Rasmussen, S. (2019). Sleep problem, suicide and self-harm in university students: a systematic review. Sleep Med. Rev. 44, 58-69. doi: 10.1016/j.smrv.2018.12.008

Sabidussi, G. (1966). The centrality index of a graph. Psychometrika 31, 581-603. doi: $10.1007 / \mathrm{bf} 02289527$

Sandman, N., Valli, K., Kronholm, E., Ollila, H. M., Revonsuo, A., Laatikainen, T., et al. (2013). Nightmares: prevalence among the finnish general adult population and war veterans during 1972-2007. Sleep 36, 1041-1050. doi: 10.5665/sleep.2806

Sandman, N., Valli, K., Kronholm, E., Revonsuo, A., Laatikainen, T., and Paunio, T. (2015). Nightmares: risk factors among the finnish general adult population. Sleep 38, 507-514. doi: 10.5665/sleep.4560
Schredl, M. (2010). Nightmare frequency and nightmare topics in a representative German sample. Eur. Arch. Psychiatry Clin. Neurosci. 260, 565-570. doi: 10. 1007/s00406-010-0112-3

Schredl, M., and Hofmann, F. (2003). Continuity between waking activities and dream activities. Conscious Cogn. 12, 298-308. doi: 10.1016/s1053-8100(02) 00072-7

Siclari, F., Baird, B., Perogamvros, L., Bernardi, G., LaRocque, J. J., Riedner, B., et al. (2017). The neural correlates of dreaming. Nat. Neurosci. 20, 872-878. doi: $10.1038 / \mathrm{nn} .4545$

Silge, J., and Robinson, D. (2016). tidytext: text mining and analysis using tidy data principles in R. J. Open Source Softw. 1:37. doi: 10.21105/joss.00037

Solomonova, E., Stenstrom, P., Paquette, T., and Nielsen, T. (2015). Different temporal patterns of memory incorporations into dreams for laboratory and virtual reality experiences: relation to dreamed locus of control. Int. J. Dream Res. 8, 10-26.

Stickgold, R., Malia, A., Maguire, D., Roddenberry, D., and O'Connor, M. (2000). Replaying the game: hypnagogic images in normals and amnesics. Science 290, 350-353. doi: 10.1126/science.290.5490.350

Tempesta, D., Socci, V., De Gennaro, L., and Ferrara, M. (2018). Sleep and emotional processing. Sleep Med. Rev. 40, 183-195. doi: 10.1016/j.smrv.2017. 12.005

Valente, T. W., and Foreman, R. K. (1998). Integration and radiality: measuring the extent of an individual's connectedness and reachability in a network. Soc. Networks 20, 89-105. doi: 10.1016/s0378-8733(97)00007-5

Waterman, D., Elton, M., and Kenemans, J. L. (1993). Methodological issues affecting the collection of dreams. J. Sleep Res. 2, 8-12. doi: 10.1111/j.13652869.1993.tb00053.x

Windt, J. M. (2010). The immersive spatiotemporal hallucination model of dreaming. Phenomenol. Cogn. Sci. 9, 295-316. doi: 10.1007/s11097-010-9163-1

World Health Organization. (2020). WHO Director-General's Opening Remarks at the Media Briefing on COVID-19-11 March 2020. Geneva: World Health Organization.

Xiao, H., Zhang, Y., Kong, D., Li, S., and Yang, N. (2020). Social capital and sleep quality in individuals who self-isolated for 14 days during the Coronavirus disease 2019 (COVID-19) outbreak in January 2020 in China. Med. Sci. Monit. 26:e923921.

Yang, Z., Algesheimer, R., and Tessone, C. J. (2016). A comparative analysis of community detection algorithms on artificial networks. Sci. Rep. 6:30750.

Zadra, A., and Donderi, D. C. (2000). Nightmares and bad dreams: their prevalence and relationship to well-being. J. Abnorm. Psychol. 109, 273-281. doi: 10.1037/ 0021-843x.109.2.273

Zhou, J., Liu, L., Xue, P., Yang, X., and Tang, X. (2020). Mental health response to the COVID-19 outbreak in China. Am. J. Psychiatry 177, 574-575. doi: 10.1176/appi.ajp.2020.20030304

Conflict of Interest: The authors declare that the research was conducted in the absence of any commercial or financial relationships that could be construed as a potential conflict of interest.

Copyright (c) 2020 Pesonen, Lipsanen, Halonen, Elovainio, Sandman, Mäkelä, Antila, Béchard, Ollila and Kuula. This is an open-access article distributed under the terms of the Creative Commons Attribution License (CC BY). The use, distribution or reproduction in other forums is permitted, provided the original author(s) and the copyright owner(s) are credited and that the original publication in this journal is cited, in accordance with accepted academic practice. No use, distribution or reproduction is permitted which does not comply with these terms. 